\title{
Local orientational order in self-assembled nanoparticle films: the role of ligand composition and salt
}

\author{
Felix Lehmkühler, Florian Schulz, Martin A. Schroer, Lara Frenzel, Holger \\ Lange and Gerhard Grübel
}

J. Appl. Cryst. (2019). 52, 777-782

\section{IUCr Journals CRYSTALLOGRAPHY JOURNALS ONLINE}

Copyright (C) International Union of Crystallography

Author(s) of this article may load this reprint on their own web site or institutional repository provided that this cover page is retained. Republication of this article or its storage in electronic databases other than as specified above is not permitted without prior permission in writing from the IUCr.

For further information see http://journals.iucr.org/services/authorrights.html 
JOURNAL OF

APPLIED

CRYSTALLOGRAPHY

ISSN 1600-5767

Received 18 February 2019

Accepted 24 May 2019

Edited by S. Sasaki, Tokyo Institute of

Technology, Yokohama, Japan

Keywords: X-ray cross-correlation analysis; selfassembly; small-angle $\mathrm{X}$-ray scattering; colloids.

\section{Local orientational order in self-assembled nanoparticle films: the role of ligand composition and salt}

\author{
Felix Lehmkühler, ${ }^{\mathrm{a}, \mathrm{b} *}$ Florian Schulz, ${ }^{\mathrm{b}, \mathrm{c}}$ Martin A. Schroer, ${ }^{\mathrm{d}}$ Lara Frenzel, ${ }^{\mathrm{a}, \mathrm{b}}$ Holger \\ Lange $^{b, c}$ and Gerhard Grübel ${ }^{a, b}$
}

\begin{abstract}
${ }^{\mathbf{a}}$ Deutsches Elektronen-Synchrotron (DESY), Notkestrasse 85, 22607 Hamburg, Germany, ${ }^{\mathbf{b}}$ The Hamburg Centre for Ultrafast Imaging, Luruper Chaussee 149, 22761 Hamburg, Germany, 'Institute of Physical Chemistry, University of Hamburg, Grindelallee 117, 20146 Hamburg, Germany, and 'European Molecular Biology Laboratory EMBL c/o DESY, Notkestrasse 85, 22607 Hamburg, Germany. *Correspondence e-mail: felix.lehmkuehler@desy.de
\end{abstract}

An X-ray cross-correlation study of the local orientational order in selfassembled films made from PEGylated gold nanoparticles is presented. The local structure of this model system is dominated by four- and sixfold order. Coadsorption of shorter ligands in the particle's ligand layer and variation of salt concentration in the suspension prior to self-assembly result in a change of local orientational order. The degree of sixfold order is reduced after salt addition. This decrease of order is less pronounced for the fourfold symmetry. The results presented here suggest complex symmetry-selective order formation upon ligand exchange and salt addition and demonstrate the versatility of X-ray crosscorrelation methods for nanoparticle superlattices.

\section{Introduction}

Nanoparticle (NP) superlattices are periodic arrays of NPs. Such assemblies can exhibit exciting new collective properties, different from those of the superposition of the individual NPs or the corresponding bulk materials, e.g. exceptional mechanical properties (Dreyer et al., 2016). Understanding and controlling NP assembly thus allow for the design of new materials and their integration into devices by various coating, casting or printing techniques (Vogel et al., 2015; Yang et al., 2016; Wu, 2017). However, the assembly of NPs is nontrivial because of the complex interplay of interparticle forces during the self-organization (Xu et al., 2013; Batista et al., 2015). In particular, the collective behavior of the stabilizing ligands on the NPs involves many interdependent processes, such as grafting layer transitions, stabilizer entropy, faceting and solvent structuring (Zeng et al., 2016).

For a general understanding of the self-organization, defined model systems are required that can be characterized in detail. An established platform to study the self-assembly is gold NPs (AuNPs) stablized with a shell of poly(ethylene glycol) (PEG) (Bishop et al., 2009; Zhang et al., 2017; Schroer et al., 2018). The high level of flexibility and control regarding size, size dispersity, and colloidal and chemical stability in combination with the high contrast in transmission electron microscopy (TEM) and X-ray scattering makes them versatile for fundamental studies (Schulz et al., 2013, 2016). Their suitability for inkjet printing was demonstrated recently (Su et al., 2019).

Regarding the investigation of three-dimensional selforganization, a specific challenge for the analytical methods is 
the requirement for obtaining structural information on bulk materials. There, electron microscopy and spectroscopy are limited because of low penetration depths. X-ray and light diffraction studies typically average ensembles and lack spatial information. X-ray cross-correlation analysis (XCCA), also known as fluctuation X-ray scattering, has been shown to overcome this limitation. Recently, the potential of XCCA has been demonstrated by various theoretical and simulation studies on 2D systems (Altarelli et al., 2010; Kurta et al., 2012; Lehmkühler et al., 2014; Malmerberg et al., 2015; Latychevskaia et al., 2015; Martin, 2017; Lhermitte et al., 2017) and shown experimentally for thin polymer systems and NPs (Gutt et al., 2014; Schroer et al., 2015; Liu et al., 2017; Lehmkühler et al., 2018), liquid crystals (Zaluzhnyy, Kurta, Sulyanova et al., 2017), and colloids and nanocrystals (Mendez et al., 2014; Mancini et al., 2016; Lehmkühler et al., 2016; Schroer, Westermeier et al., 2016; Mendez et al., 2016; Zaluzhnyy, Kurta, André et al., 2017; Mancini et al., 2018). We previously measured the local orientational order of self-assembled NP films of thicknesses of up to a few micrometres by means of XCCA (Schroer et al., 2015; Lehmkühler et al., 2018).

In the present work, we apply XCCA to self-assembled AuNP films to demonstrate the strong sensitivity of the local orientational order on the NP's surface chemistry, and also on the salinity of the solvent present during the self-organization. XCCA proves to be a powerful tool to characterize NP superlattices and can contribute to the optimization of largescale structures.

\section{Experimental}

\subsection{Sample preparation}

Quasi-spherical gold nanoparticles (diameter $d=27.7 \pm$ $2.1 \mathrm{~nm}$ ) were synthesized with a seeded growth protocol (Bastús et al., 2011). The ligand $\alpha$-methoxypoly(ethylene glycol)- $\omega$-(11-mercaptoundecanoate) (PEGMUA) with $M \simeq$ $2000 \mathrm{~g} \mathrm{~mol}^{-1}$ was synthesized as described previously (Schulz et al., 2013). The syntheses of AuNP coated with PEGMUA (AuNP@PEGMUA) or 1:1 mixed ligand layers of PEGMUA and 11-mercaptoundecanoic acid (MUA) were conducted as described earlier for smaller AuNPs (Schulz et al., 2016). An as-synthesized aqueous solution of citrate-stabilized AuNP $[c(\mathrm{AuNP})=1 \mathrm{n} M, 100 \mathrm{ml}]$ was added under rapid stirring to PEGMUA or a 1:1 ligand mixture of PEGMUA and MUA in ethanol to yield $80 \mu M$ of ligands in total in the final mixture at room temperature. After several hours of stirring, the AuNP conjugates were purified and concentrated by repeated centrifugations $(6000 \mathrm{~g}$ for $30 \mathrm{~min}$ ) to $200 \mathrm{n} M$. The AuNP concentrations were determined by UV/vis spectroscopy (Haiss et al., 2007); the diameter was obtained by TEM analysis. Absorbance measurements were carried out using a Varian Cary 50 spectrometer. UV microcuvettes sealed with lids (Plastibrand, Carl Roth $\mathrm{GmbH}$, Karlsruhe, Germany) were used for all experiments. The concentrated AuNPconjugate solutions are colloidally stable and can be dried and redispersed on various substrates without aggregation.
We prepared thin films assembled from different AuNP dispersions: (1) AuNP@PEGMUA, (2) mixed ligands of 1:1 PEGMUA and MUA (denoted as 50\% MUA), (3) AuNP@PEGMUA with $5 \mathrm{mM} \mathrm{NaCl}$, and (4) $50 \%$ MUA dispersions with $5 \mathrm{~m} M \mathrm{NaCl}$. The nanoparticle films were prepared on square silicon nitride membranes in square silicon supporting frames (Silson Ltd, UK). The membrane area was $3.0 \times$ $3.0 \mathrm{~mm}$ with a thickness of $1 \mu \mathrm{m}$. The frame size was $7.5 \times$ $7.5 \mathrm{~mm}$ and the frame thickness was $535 \mu \mathrm{m}$. Under these conditions the solutions dry in a well defined volume and the film thickness can be controlled by the AuNP concentration. This way, film thicknesses between approximately 100 and $600 \mathrm{~nm}$ are obtained (Lehmkühler et al., 2018). For each film, $7.5 \mu \mathrm{l}$ solutions of AuNP with the different ligands and salt content described above were pipetted onto the framed membrane and dried at $338 \mathrm{~K}$.

\subsection{X-ray scattering experiments}

The X-ray scattering experiments were performed on the coherence applications beamline P10 at PETRA III (DESY, Hamburg). We used the GINIX setup in small-angle scattering geometry (Kalbfleisch et al., 2011) in order to achieve beam sizes of $400 \times 400 \mathrm{~nm}$ by a Kirkpatrick-Baez mirror system. The X-ray photon energy was set to $E=13.8 \mathrm{keV}$. As detector, a Dectris Eiger $X 4 \mathrm{M}$ was placed $5 \mathrm{~m}$ downstream of the sample.

After the samples had been put into the sample holder, representative regions of interest (ROIs) were defined by optical microscopy. Scattering patterns were taken from these ROIs in grids of typically $30 \times 30 \mu \mathrm{m}(x \times y)$ in steps of $500 \mathrm{~nm}$ and with $1 \mathrm{~s}$ exposure time per pattern. At least four ROIs were measured for each sample, which results in ensembles of more than 15000 individual scattering patterns per sample. The patterns were corrected for scattering background from the empty chamber and SiN membranes. Afterwards, XCCA was applied following the procedures discussed elsewhere (Lehmkühler et al., 2014).

\section{Results}

An example of a scattering pattern is shown in Fig. 1(a). The beamstop, beamstop holder and inactive detector areas have been masked. The pattern suggests a sixfold local orientational order as expressed by the six strong reflections at the maximum of $I(q)$. This agrees with our previous study, where we reported a well developed four- and sixfold local orientational order in such thin films (Lehmkühler et al., 2018).

The azimuthally integrated intensity $I(q)$ of all samples is shown in Fig. 1(b). As the sample preparation procedure results in heterogeneous films of different thicknesses on a local scale (Lehmkühler et al., 2018), I( $q$ ) has been averaged for regions of similar thickness of all samples, as expressed by the average intensity from each pattern. All $I(q)$ show a well developed structure factor peak around $q=0.21-0.24 \mathrm{~nm}^{-1}$; however, its amplitude differs for all samples. A strong peak can be observed for the AuNPs with PEGMUA ligands, which 

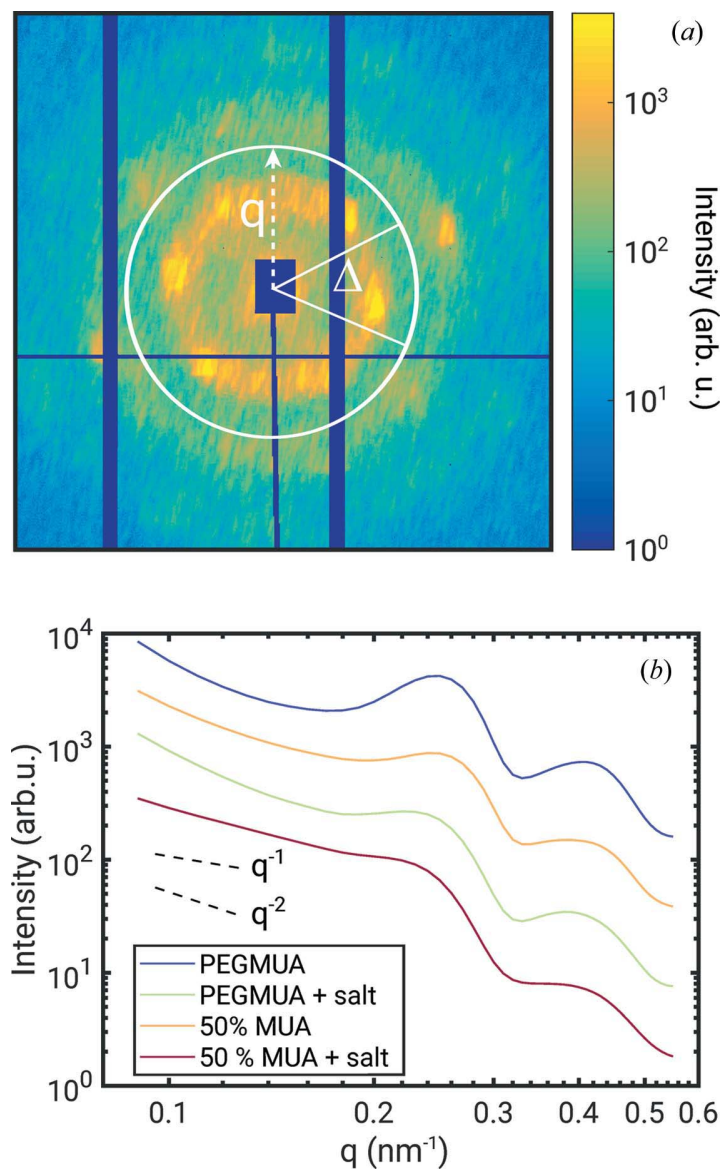

Figure 1

(a) Example of a diffraction pattern taken from the PEG sample. The modulus $q$ and the azimuthal difference $\Delta$ are indicated. (b) Integrated intensity $I(q)$ for all samples studied. The data are offset vertically for clarity.

is less pronounced upon addition of salt to the solvent prior to self-assembly. The same trend can be observed for the $50 \%$ MUA ligands, which show less prominent peaks compared with the corresponding PEGMUA samples. This suggests that (i) the addition of salt and (ii) the presence of MUA in the particles' shell lead to less ordered films. The latter has been observed in assembled monolayers (Schulz et al., 2016); however, the effect of MUA on the assembly of thin 3D films has not been studied so far. In addition, for $50 \%$ MUA + salt the forward scattering is increased, suggesting the formation of disordered clusters of different shape, e.g. rod-like assemblies. A fit of $I(q)$ for $q \leq$ $0.18 \mathrm{~nm}^{-1}$ yields a power-law dependence of $I(q) \propto q^{-1.6}$, which resembles the exponents expected for differently shaped quasi-2D aggregates of between -1 and -2 , shown as dashed lines in Fig. 1(b) (de Jeu, 2016). Such aggregates may originate from the formation of cracks during drying and are in addition visible by scanning electron microscopy as shown previously (Lehmkühler et al., 2018).

In order to obtain quantitative information on the degree of order upon self-assembly, the data were analyzed in the framework of XCCA. XCCA experiments probe the orientational order of the sample via angular correlation functions. In our case we calculate correlations of intensity $I(q, \phi)$ at fixed wavevector transfer $q=(4 \pi / \lambda) \sin (\theta / 2)$, with the X-ray wavelength $\lambda$, the scattering angle $\theta$ and azimuthal angle $\phi$. The correlation function of intensity at angular differences $\Delta$ is thus given by (Wochner et al., 2009)

$$
C(q, \Delta)=\frac{\langle I(q, \phi) I(q, \phi+\Delta)\rangle_{\phi}-\langle I(q, \phi)\rangle_{\phi}^{2}}{\langle I(q, \phi)\rangle_{\phi}^{2}} .
$$

The type and degree of local orientational order are expressed by the Fourier coefficients of $\ell$ th order $\hat{C}_{\ell}(q)$ of $C(q)$ with respect to $\Delta$ (Altarelli et al., 2010; Kurta et al., 2012; Lehmkühler et al., 2014; Kurta et al., 2016). Applying the WienerKhinchin theorem, these are given by the Fourier coefficients $\hat{I}_{\ell}(q)$ of normalized intensity via

$$
\hat{C}_{\ell}(q)=\left|\hat{I}_{\ell}(q)\right|^{2} .
$$

To obtain access to the ensemble-average orientational order of the sample, the variance

$$
\Psi_{\ell}(q)=\left\langle\hat{I}_{\ell}(q)^{2}\right\rangle_{\mathrm{e}}-\left\langle\hat{I}_{\ell}(q)\right\rangle_{\mathrm{e}}^{2}
$$

has been introduced (Lehmkühler et al., 2014), where \langle\rangle$_{\mathrm{e}}$ denotes the average over the studied ensemble.

In our previous study (Lehmkühler et al., 2018) we reported an increase of orientational order with sample thickness. For thin films the scattered intensity is proportional to the sample thickness in small-angle X-ray scattering (SAXS) experiments. So that we can neglect the impact of sample thickness on the XCCA results, only patterns with a similar average intensity and thus similar film thickness were used for the analysis.

The ensemble averages $\Psi_{\ell}(q)$ are shown in Fig. 2 for $\ell \leq 20$ as a function of $q$ and $\ell$. The results have been averaged over
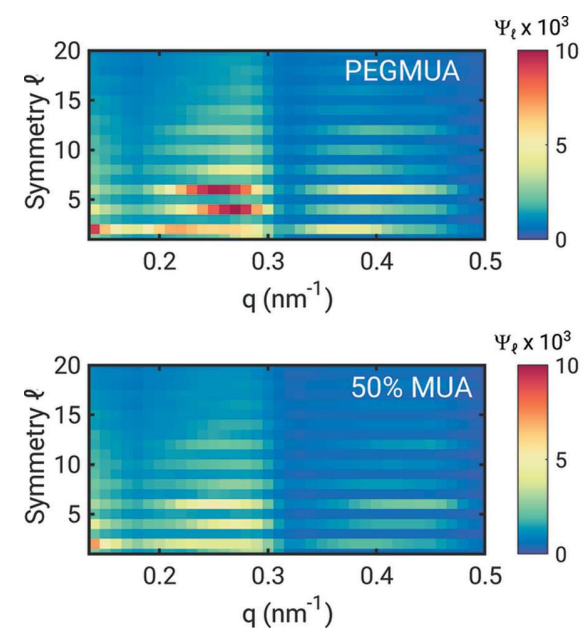

Figure 2

Orientational order $\Psi_{\ell}(q)$ for all studied samples.
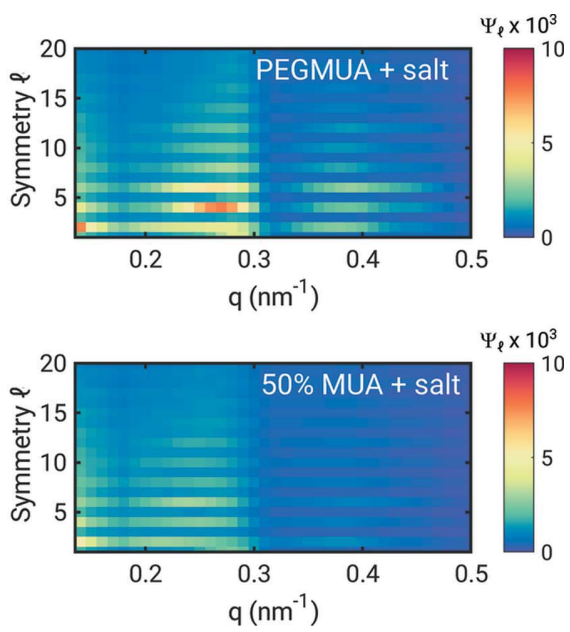


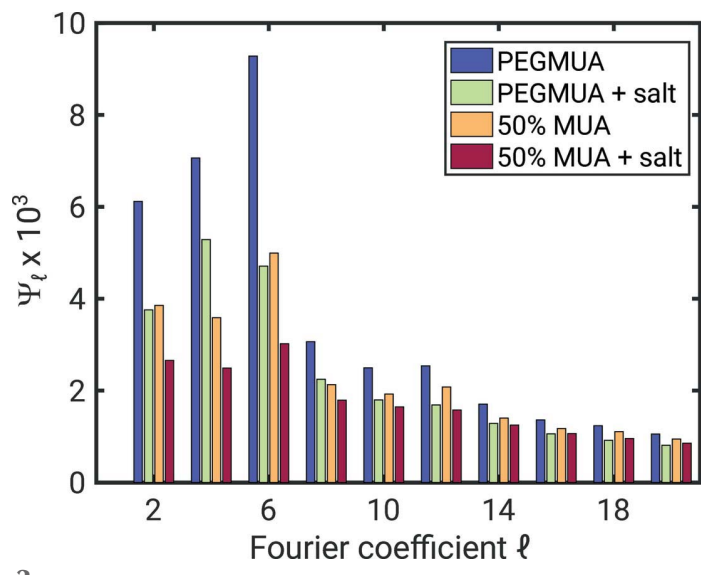

Figure 3

Degree of orientational order $\Psi_{\ell}$ for $q=0.24 \mathrm{~nm}^{-1}$.

all studied sample spots. Only even coefficients contribute significantly, which underlines the validity of Friedel's law for the thin samples (Lehmkühler et al., 2014; Liu et al., 2016). Comparing all samples, the PEGMUA sample shows the highest amplitudes, followed by PEGMUA with salt, $50 \%$ MUA and 50\% MUA with salt. Similarly to our previous study (Lehmkühler et al., 2018), the PEGMUA samples show dominant four- and sixfold symmetries. This is less pronounced for the 50\% MUA samples, suggesting the formation of less ordered films. Furthermore, the highest degree of $\Psi_{\ell}$ can be found around $q=0.25 \mathrm{~nm}^{-1}$, i.e. in the vicinity of the maximum of $I(q)$.

The degree of orientational order $\Psi_{\ell}$ at $q=0.24 \mathrm{~nm}^{-1}$ is displayed in Fig. 3. As discussed above, dominant four- and sixfold symmetries are present for all samples. For $\ell \geq 8$, the orientational order $\Psi_{\ell}$ is similar for all samples, with slightly larger values for PEGMUA. $\Psi_{4}$ and $\Psi_{6}$ show the discussed trend, i.e. $\Psi($ PEGMUA $)>\Psi($ PEGMUA + salt $) \simeq \Psi(50 \%$ MUA $)>\Psi(50 \%$ MUA + salt $)$. The amplitude changes by a factor of about 2-3 between PEGMUA and 50\% MUA + salt. For the latter, the amplitude of $\Psi_{\ell}$ varies only slightly with $\ell$, suggesting less preferred local orientational order as present in the other samples.

In order to study the orientational order in more detail, the $q$ dependence of $\Psi_{6}$ is shown in Fig. 4(a). Note that the asymptotic behavior is similar for other even values of $\ell$, as can be seen from Fig. 2. In general, $\Psi_{\ell}$ was found to show an asymptotic $q^{-2}$ behavior, best developed for $\ell=4$ and $\ell=6$. In contrast, Malmerberg et al. (2015) reported a $q^{-8}$ dependence of the coefficients. This should thus asymptotically approach a constant value by normalization to $I(q)^{2}$ as done in equation (1), because $I(q) \propto q^{-4}$ for spheres. We assign the remaining $q$ dependence to a particular fingerprint of existing orientational order, because it is less defined for weak even and odd $\ell$.

While the general $q$ dependence is similar for all samples, the results differ in amplitude between approximately $10^{-2}$ for PEGMUA and $3 \times 10^{-3}$ for $50 \%$ MUA with salt at $q=$ $0.25 \mathrm{~nm}^{-1}$.

As discussed in earlier publications (Lehmkühler et al., 2014, 2016, 2018) and shown in Fig. 2, the odd coefficients do
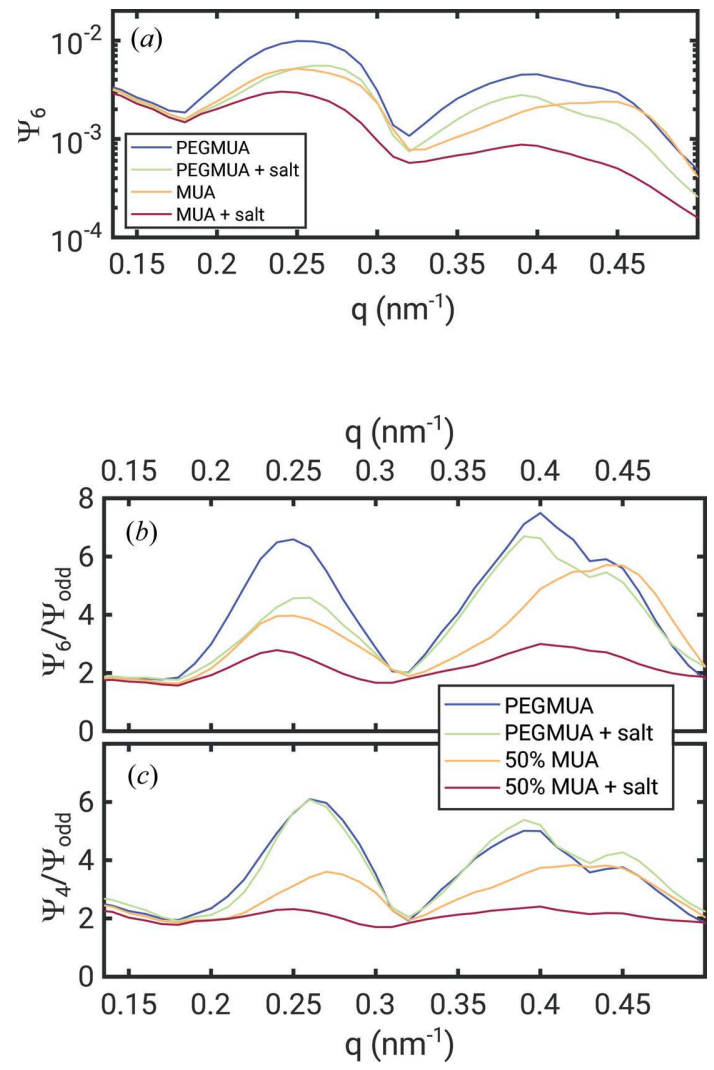

Figure 4

(a) $q$ dependence of $\Psi_{6}$ for all studied samples. (b) $\Psi_{6}$ normalized to the average of odd coefficients between $\ell=3$ and $\ell=19$. (c) Same as $(b)$ for $\Psi_{4}$.

not differ significantly and represent basically a background measure, e.g. reflecting noise and setup-dependent contributions (Lehmkühler et al., 2014). They may also originate from the curvature of the Ewald sphere (Liu et al., 2016), which is otherwise negligible for the thin samples studied here. Moreover, they account for intensity variations between different sample systems (Lehmkühler et al., 2018). Therefore, we normalize $\Psi_{6}$ and $\Psi_{4}$ to the average $\Psi_{\text {odd }}$ of the odd coefficients between $\ell=3$ and $\ell=19$. The results are shown in Figs. $4(b)$ and $4(c)$. For $\Psi_{6}$ the results resemble the observation from Figs. 3 and $4(a)$. In addition, a splitting of the second peak around $q=0.4 \mathrm{~nm}^{-1}$ becomes obvious for the PEGMUA samples, which is absent for $50 \%$ MUA. This is accompanied by a peak shift to larger $q$ for $50 \%$ MUA. We associate this with the thinner effective ligand layer upon addition of MUA (Schulz et al., 2016), which affects the length scales of order formation. These observations are mirrored for $\Psi_{4}$; however, its amplitudes are slightly lower than those of $\Psi_{6}$. Most interestingly, the results for PEGMUA and PEGMUA + salt match each other. This indicates that the addition of salt leads to a reduced degree of sixfold order, while the fourfold structure remains unaffected.

\section{Summary and conclusions}

We have studied the structure of self-assembled films of gold nanoparticles as a function of PEG-based ligand composition 
and salt content in the colloidal dispersion. The SAXS data suggest a decrease of order upon addition of MUA to the ligand shell as well as upon the addition of salt. The orientational order studied by XCCA is governed by four- and sixfold order for both types of ligands. This agrees with our previous study, where heterogeneous films with cubic and hexagonal local orientational order were obtained (Lehmkühler et al., 2018). The comparison between PEGMUA and 50\% MUA indicates that the presence of MUA in the ligand shell results in less ordered self-assembled films. This is in agreement with previous observations of monolayers, where particles with pure PEGMUA ligands form a hexatic 2D film whose order is disturbed when MUA is present (Schulz et al., 2016). It is related to different surface charges of PEGMUA-coated AuNPs and those with mixed ligand shells affecting the assembly of monolayers. The addition of salt results in a similar effect for both samples, i.e. a significant decrease of the degree of orientational order. In addition, MUA addition leads to different ligand layer conformations that affect the stability of the particles (Schulz et al., 2018). Obviously, this way the assembly of layers in the (sub-)micrometre range as studied in this work is affected as well.

Addition of salt decreases the solubility of PEG in water (Ren et al., 2011). Recently, the formation of supercrystals of AuNP@PEG in solution at high salt concentrations was observed (Zhang et al., 2017; Schroer et al., 2018). This suggests the formation of well ordered self-assembled structures after addition of salt to the dispersion. In contrast to those observations, the degree of orientational order decreases for the salt samples, which is accompanied with a less defined averaged structure obtained by SAXS. In particular for the 50\% MUA sample, the increased forward scattering suggesting the formation of disordered clusters is reflected by the low degree of orientational order obtained by XCCA.

For samples with PEGMUA ligands, the degree of sixfold symmetry expressed by $\Psi_{6}(q)$ is reduced by the addition of salt. In contrast, $\Psi_{4}(q)$ remains unchanged. Most importantly, samples with 50\% MUA ligands show a reduced orientational order for all $q$ upon salt addition. This suggests complex symmetry-selective effects of salt and ligand exchange on the formation of self-assembled superstructures during the drying process. Recently we observed changes of the compressibility of PEG-based ligands after salt addition as well as after ligand modification with MUA (Schroer, Schulz et al., 2016; Schulz et al., 2018; Schroer et al., 2018). Therefore, the plasticity of ordered and ordering clusters may change during drying, as reported recently for shear band formation in directed drying processes (Yang et al., 2018). This may further lead to modifications of local orientational order as reported in this study.

In summary, we have analyzed the role of ligand layer composition and salt addition for the local orientational order of self-assembled AuNP@PEG films with XCCA. PEG and other polymer coatings allow one to stabilize NPs effectively and to tune their properties but will also affect their selfassembly behavior. Detailed structural analyses of such materials will contribute to the understanding of NP assembly, thus assisting the design of materials and devices based on NP films.

\section{Acknowledgements}

Parts of this research were carried out at PETRA III at DESY, a member of the Helmholtz Association (HGF). We would like to thank Markus Osterhoff (Institute of X-ray Physics, Göttingen University) and Michael Sprung (DESY) for assistance in using the GINIX setup on beamline P10.

\section{Funding information}

This work is supported by the Cluster of Excellence 'The Hamburg Centre for Ultrafast Imaging' of the Deutsche Forschungsgemeinschaft (DFG) - EXC 1074 - project ID 194651731. FS acknowledges the Deutsche Forschungsgemeinschaft (grant No. SCHU 3019/2-1). MAS acknowledges the Röntgen-Ångström cluster project TTSAS (Bundesministerium für Bildung und Forschung project No. 05K16YEA) for financial support.

\section{References}

Altarelli, M., Kurta, R. \& Vartanyants, I. (2010). Phys. Rev. B, 82, 104207.

Bastús, N. G., Comenge, J. \& Puntes, V. (2011). Langmuir, 27, 1109811105.

Bishop, K. J. M., Wilmer, C. E., Soh, S. \& Grzybowski, B. A. (2009). Small, 5, 1600-1630.

Dreyer, A., Feld, A., Kornowski, A., Yilmaz, E. D., Noei, H., Meyer, A., Krekeler, T., Jiao, C., Stierle, A., Abetz, V., Weller, H. \& Schneider, G. A. (2016). Nat. Mater. 15, 522-528.

Gutt, C., Grodd, L., Mikayelyan, E., Pietsch, U., Kline, R. J. \& Grigorian, S. (2014). J. Phys. Chem. Lett. 5, 2335-2339.

Haiss, W., Thanh, N. T. K., Aveyard, J. \& Fernig, D. G. (2007). Anal. Chem. 79, 4215-4221.

Jeu, W. H. de (2016). Basic X-ray Scattering for Soft Matter. Oxford University Press.

Kalbfleisch, S., Neubauer, H., Krüger, S. P., Bartels, M., Osterhoff, M., Mai, D. D., Giewekemeyer, K., Hartmann, B., Sprung, M. \& Salditt, T. (2011). AIP Conf. Proc. 1365, 96-99.

Kurta, R. P., Altarelli, M. \& Vartanyants, I. A. (2016). Adv. Chem. Phys. 161, 1.

Kurta, R. P., Altarelli, M., Weckert, E. \& Vartanyants, I. A. (2012). Phys. Rev. B, 85, 184204.

Latychevskaia, T., Mancini, G. F. \& Carbone, F. (2015). Sci. Rep. 5, 16573.

Lehmkühler, F., Fischer, B., Müller, L., Ruta, B. \& Grübel, G. (2016). J. Appl. Cryst. 49, 2046-2052.

Lehmkühler, F., Grübel, G. \& Gutt, C. (2014). J. Appl. Cryst. 47, 1315-1323.

Lehmkühler, F., Schulz, F., Schroer, M. A., Frenzel, L., Lange, H. \& Grübel, G. (2018). IUCrJ, 5, 354-360.

Lhermitte, J. R., Tian, C., Stein, A., Rahman, A., Zhang, Y., Wiegart, L., Fluerasu, A., Gang, O. \& Yager, K. G. (2017). J. Appl. Cryst. 50, 805-819.

Liu, A. C. Y., Tabor, R. F., Bourgeois, L., de Jonge, M. D., Mudie, S. T. \& Petersen, T. C. (2016). J. Stat. Mech. Theory Exp. 2016, 054046 . 
Liu, A. C. Y., Tabor, R. F., de Jonge, M. D., Mudie, S. T. \& Petersen, T. C. (2017). Proc. Natl Acad. Sci. USA, https://doi.org/10.1073/ pnas.1707198114.

Malmerberg, E., Kerfeld, C. A. \& Zwart, P. H. (2015). IUCrJ, 2, 309316.

Mancini, G. F., Karl, R. M., Shanblatt, E. R., Bevis, C. S., Gardner, D. F., Tanksalvala, M. D., Russell, J. L., Adams, D. E., Kapteyn, H. C., Badding, J. V., Mallouk, T. E. \& Murnane, M. M. (2018). Opt. Express, 26, 11393-11406.

Mancini, G. F., Latychevskaia, T., Pennacchio, F., Reguera, J., Stellacci, F. \& Carbone, F. (2016). Nano Lett. 16, 2705-2713.

Martin, A. V. (2017). IUCrJ, 4, 24-36.

Mendez, D., Lane, T. J., Sung, J., Sellberg, J., Levard, C., Watkins, H., Cohen, A. E., Soltis, M., Sutton, S., Spudich, J., Pande, V., Ratner, D. \& Doniach, S. (2014). Philos. Trans. R. Soc. B, 369, 20130315.

Mendez, D., Watkins, H., Qiao, S., Raines, K. S., Lane, T. J., Schenk, G., Nelson, G., Subramanian, G., Tono, K., Joti, Y., Yabashi, M., Ratner, D. \& Doniach, S. (2016). IUCrJ, 3, 420-429.

Ren, C., Tian, W., Szleifer, I. \& Ma, Y. (2011). Macromolecules, 44, 1719-1727.

Schroer, M. A., Gutt, C., Lehmkühler, F., Fischer, B., Steinke, I., Westermeier, F., Sprung, M. \& Grübel, G. (2015). Soft Matter, 11, 5465-5472.

Schroer, M. A., Lehmkühler, F., Möller, J., Lange, H., Grübel, G. \& Schulz, F. (2018). J. Phys. Chem. Lett. 9, 4720-4724.

Schroer, M. A., Schulz, F., Lehmkühler, F., Möller, J., Smith, A. J., Lange, H., Vossmeyer, T. \& Grübel, G. (2016). J. Phys. Chem. C, 120, 19856-19861.

Schroer, M. A., Westermeier, F., Lehmkühler, F., Conrad, H., Schavkan, A., Zozulya, A. V., Fischer, B., Roseker, W., Sprung, M., Gutt, C. \& Grübel, G. (2016). J. Chem. Phys. 144, 084903.

Schulz, F., Dahl, G. T., Besztejan, S., Schroer, M. A., Lehmkühler, F., Grübel, G., Vossmeyer, T. \& Lange, H. (2016). Langmuir, 32, $7897-$ 7907.
Schulz, F., Möller, J., Lehmkühler, F., Smith, A. J., Vossmeyer, T., Lange, H., Grübel, G. \& Schroer, M. A. (2018). Part. Part. Syst. Charact. 35, 1700319.

Schulz, F., Vossmeyer, T., Bastús, N. G. \& Weller, H. (2013). Langmuir, 29, 9897-9908.

Silvera Batista, C. A., Larson, R. G. \& Kotov, N. A. (2015). Science, 350, 1242477.

Su, C.-H., Chiu, H.-L., Chen, Y.-C., Yesilmen, M., Schulz, F., Ketelsen, B., Vossmeyer, T. \& Liao, Y.-C. (2019). Langmuir, 35, 32563264.

Vogel, N., Retsch, M., Fustin, C.-A., Del Campo, A. \& Jonas, U. (2015). Chem. Rev. 115, 6265-6311.

Wochner, P., Gutt, C., Autenrieth, T., Demmer, T., Bugaev, V., Ortiz, A., Duri, A., Zontone, F., Grübel, G. \& Dosch, H. (2009). Proc. Natl Acad. Sci. USA, 106, 11511-11514.

Wu, W. (2017). Nanoscale, 9, 7342-7372.

Xu, L., Ma, W., Wang, L., Xu, C., Kuang, H. \& Kotov, N. A. (2013). Chem. Soc. Rev. 42, 3114-3126.

Yang, B., Smith, N. D., Johannes, A., Burghammer, M. \& Smith, M. I. (2018). Sci. Rep. 8, 12979.

Yang, J., Choi, M. K., Kim, D.-H. \& Hyeon, T. (2016). Adv. Mater. 28, 1176-1207.

Zaluzhnyy, I. A., Kurta, R. P., André, A., Gorobtsov, O. Y., Rose, M., Skopintsev, P., Besedin, I., Zozulya, A. V., Sprung, M., Schreiber, F., Vartanyants, I. A. \& Scheele, M. (2017). Nano Lett. 17, 35113517.

Zaluzhnyy, I. A., Kurta, R. P., Sulyanova, E. A., Gorobtsov, O. Y., Shabalin, A. G., Zozulya, A. V., Menushenkov, A. P., Sprung, M., Krówczyński, A., Górecka, E., Ostrovskii, B. I. \& Vartanyants, I. A. (2017). Soft Matter, 13, 3240-3252.

Zeng, C., Chen, Y., Kirschbaum, K., Lambright, K. J. \& Jin, R. (2016). Science, 354, 1580-1584.

Zhang, H., Wang, W., Akinc, M., Mallapragada, S., Travesset, A. \& Vaknin, D. (2017). Nanoscale, 9, 8710-8715. 